\title{
Defect detection in ceramic materials by quantitative infrared thermography.
}

\author{
by G. M. Revel* and S. Rocchi*
}

*Dipartimento di Meccanica - Università Politecnica delle Marche, Ancona, Italy

\begin{abstract}
In this paper infrared (IR) measurements and dedicated signal processing algorithms were developed and applied for defect detection on green ceramic tiles. Tiles with defects having known dimension and position were realized and tested by acquiring either heating and cooling transients of the tile subjected to thermal pulse excitation. As rarely defects appear directly in the thermal map, post-processing algorithms were used. Satisfactory results were achieved employing thermal tomography (TT), pulsed phase thermography (PPT), principal component analysis (PCA) and techniques of image balancing and trend removing. The performances of the different strategies were quantitatively compared in terms of contrast and signal-to-noise ratio.
\end{abstract}

\section{Introduction}

In the ceramic industry rarely testing systems were employed to on-line detect the presence of defects in ceramic tiles. Defects in the ceramic body [1,2] are usually originated during the pressing stage due to incorrect process parameters or not controlled raw materials. These defects are generally characterized by delaminations, inclusion of heterogeneous material or agglomerates, which decrease the structural strength.

The high cost of possible inspection systems (as X-ray methods) able to detect information about the internal structure of ceramic material, if compared with the low cost of the ceramic products, determines in most of the cases the choice of an empirical optimization of manufacture parameters or visual inspection techniques (only for defects involving the tile surface).

In many technological sectors, infrared (IR) inspection is utilized for damage detection, thanks to its resolution, easiness, full-field capability, in-field applicability and finally to the continuously decreasing costs of thermographic systems. Thermographic techniques [3] are commonly applied to building inspection, process control and monitoring, predictive maintenance, evaluation of material properties, medical diagnosis.

In ceramic industry some applications of thermographic systems can be found in

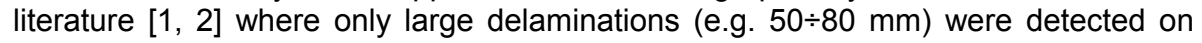
production green ceramic tiles. In fact till the tile is in a green state (i.e. pressed, but not yet dried and fired), the ceramic material is completely recyclable and then an inspection to the whole production can be very useful.

With respect to previous works, here IR inspection by thermography is focused on the development of a method to detect small defects (with a maximum dimension of $30 \mathrm{~mm}$ ) in real large production tiles, thus determining limits and performances of the technique. An active thermography approach is proposed trough pulse heating ceramic tiles and recording the temperature decay (pulsed thermography). Heating is limited to a few degrees above the initial temperature of the tile. The temperature of the material changes rapidly after the initial thermal stimulation because of the 
thermal front propagation, by diffusion, under the surface and of radiation and convection losses. The presence of a defect alters the diffusion rate so that during observation of the surface temperature, defects appear as areas of different temperatures with respect to a surrounding sound area once the thermal front has reached them [3].

As rarely small defects appear directly in the thermal maps, different post-processing algorithms were used and compared based on thermal tomography (TT), pulsed phase thermography (PPT), principal component analysis (PCA) and techniques of image balancing and trend removing. The performances of the different strategies were quantitatively compared in terms of contrast and signal-to-noise ratio.

The method can be potentially applied in production, on-line or close to the line on samples collected e.g. every 5 minutes for quality monitoring.

\section{Samples and experimental setup}

Defected green ceramic tiles were produced in the laboratory of a ceramic industry by inserting during the pressing process heterogeneous materials, as plastic pieces or row material agglomerates. The defects have known different dimension (from $5 \times 5$ $\mathrm{mm}$ to $30 \times 30 \mathrm{~mm}$ ), location and depth, in order to test the performances of the proposed methods. The tiles have dimension of $325 \times 325 \times 10 \mathrm{~mm}$ and are real production pieces at the green state, i.e. not dried and fired.

In figure 1 the experimental set-up developed to perform active thermography is shown. The structure is realized in aluminum, in such a way as to reduce the irradiation of the bench during the tests. The thermal excitation is given by four IR lamps capable to irradiate a quite uniform amount of heat (even if not perfectly uniform for a sample large $0.4 \times 0.4 \mathrm{~m}$ ) in an adjustable time interval, with an overall power of $4 \mathrm{~kW}$ (figure 1).

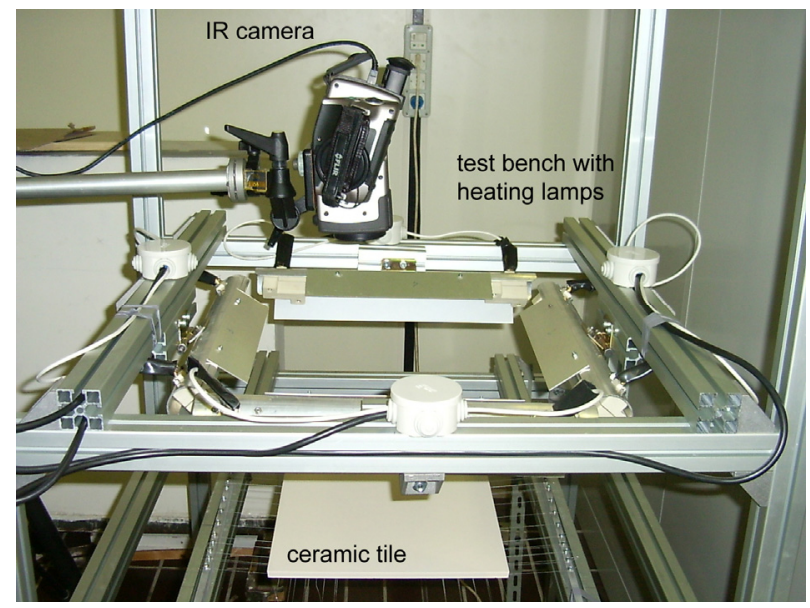

Fig. 1. Measurement setup for active thermography: IR camera and test bench equipped with four heating lamps.

Thermographic measurements were performed by a long wave infrared camera (FLIR ThermaCAM ${ }^{\mathrm{TM}}$ S40) equipped with a FPA (Focal Plane Array) of uncooled microbolometers, sensible in the spectral range from 7.5 to $13 \mu \mathrm{m}$ and characterized by an accuracy in temperature measurement of $\pm 2{ }^{\circ} \mathrm{C}$ or $\pm 2 \%$ of the reading, 
according to the producer specifications. The setup is completed by a PC controlling the IR camera trough IEEE 1394 connection and acquiring sequential thermograms. The experimental procedure consisted of heating the tile in reflection mode (i.e. lamps and infrared detector at the same side) with a heat pulse and recording the surface temperature map for a time period sufficiently long to reveal the defect and to keep a good resolution in low frequencies (see Par. 3.2); the acquisition rate chosen was $10 \mathrm{~Hz}$, starting the acquisition just before the application of the pulse.

After several tests, it was observed that the best results were obtained with an excitation of about $30 \mathrm{~s}$ and acquisition of about $90 \mathrm{~s}$. However satisfactory results can be achieved even with shorter times and this is important for the on-line application.

\section{Software and algorithms for infrared image processing}

In many cases, the raw thermal images are not able to highlight the presence of the defect. Therefore it is necessary to apply post-processing algorithms for the extraction of the defect features. In this work different approaches have been analyzed, implemented in LabVIEW and compared. The following paragraphs provide an in depth description of them.

\subsection{Thermal tomography}

Thermal tomography [3] is based on the measurement of the surface temperature on the component under inspection after the initial thermal heating.

The parameter chosen to characterize the thermal behavior of the specimen is the thermal contrast $C$ that is computed at time $t$ for a given pixel $(i, j)$ from temperature image $T$ by this equation:

$C(i, j, t)=\frac{T(i, j, t)-T\left(i, j, t_{0}\right)}{T_{d f a}(t)-T_{d f a}\left(t_{0}\right)}$

where $T_{d f a}$ corresponds to the surface temperature over a defect free area in the tile and $t_{0}$ is the initial time.

From such an acquired sequence of infrared images it is possible to compute, for every pixel, the time when the thermal contrast is maximum $\left(t_{c_{-} \max }\right)$. The distribution of $t_{c_{-} \max }$ values for all the pixels in the image is called a timegram $T G M_{c_{-} \max }$ which has the same dimensions as an infrared image.

Assuming uniform heating of the component surface, areas of the specimen having uniform thermal properties will cause the maximum thermal contrast to occur in the same time window in the timegram. On the contrary, defect or delaminations having different thermal properties exhibit different values of that parameter and consequently different time values in the timegram; this makes defect detection possible.

Disadvantages of this technique are essentially concentrated both in the sensitivity to thermal distributions on the sample, due to uneven heating, geometric features (edges of tiles exhibit higher cooling rate) or uneven emissivity, and in the need of knowledge of some defect free zones in the tile. 


\subsection{Pulsed phase thermography}

Pulsed thermography consists of pulse heating the specimen briefly and recording the surface temperature decay curve with an infrared camera.

A pulse can be decomposed into a multitude of individual sinusoidal components; therefore pulse heating of a specimen corresponds to the simultaneous launching into the specimen of thermal waves of various amplitudes and frequencies. Utilizing a thermal pulse for stimulation of the specimen, thermal waves of many different frequencies are applied simultaneously.

In the pulsed phase thermography [4] the response of the specimen on the frequency domain is extracted and analyzed.

Extraction of the various frequencies is performed by the application of FFT algorithm on each pixel of the sequence of thermal images and both amplitude and phase images are reconstructed.

With a sequence of $N$ thermal images, $N / 2$ frequency values are available and the discrete frequencies $f_{n}$ are given by this relation:

$$
f_{n}=\frac{n}{N \Delta}, \quad n=0,1, \ldots, \frac{N}{2}-1
$$

where $\Delta$ is the time interval between two consecutive thermal images.

The maximum frequency available is thus limited by the acquisition rate, while the minimum frequency is limited by the duration of the experiment.

After this procedure a sequence of thermal images is obtained in the frequency domain (amplitude and phase) and, since phase images are less affected by heating non-uniformities, experimental constraints about positions of thermal sources are relaxed. In phase images the portions of the surface above a defect exhibit different phase delays, at several frequencies, respect to the rest of surface without defect.

\subsection{Principal Component Analysis (PCA) applied to IR image sequence}

The Principal Component Analysis (PCA) [5] is a linear projection technique for converting a matrix $A$ of dimension $m \times q$ to a matrix $A_{p}$ of lower dimension $p \times q$ (with $p<m$ ) by projecting $A$ onto a new set of principal axis.

In PCA a set of correlated variables are transformed into a set of uncorrelated variables which are ordered by reducing variability. The uncorrelated variables are linear combinations of the original variables, and the last of these variables can be removed with minimum loss of real data. The main use of PCA is to reduce the dimensionality of a data set while retaining as much information as is possible; it computes a compact and optimal description of the dataset.

The main algorithm can be synthesized in these steps: data standardization (scaling and mean centering), calculation of the correlation matrix and its eigenvectors and eigenvalues, arrangement of eigenvalues from highest to lowest. The last operation gives components in order of significance because the $j^{\text {th }}$ eigenvalue quantifies the amount of the variance of the original dataset explained by the $j^{\text {th }}$ principal component.

The first principal component is then the combination of variables that explains the greatest amount of variation; the second principal component defines the next largest amount of variation and is independent from the first principal component and so on. 
There can be as many possible principal components as there are variables; if original data have $m$ dimensions, after calculation, also eigenvectors and eigenvalues are $m$; then, following some criteria to be established case by case, only the first $p$ eigenvectors can be chosen and the final dataset has only $p$ dimension. The final step regards the construction of a new dataset by projecting original data in the new reference frame created with principal components; the projection generates a significant data reduction with a condensation in $A_{p}$ of all the relevant information originally contained in $A$, with enhanced contrast respect to the noise.

In literature PCA have been employed to elaborate different kind of datasets in order to identify patterns and to highlight their similarities and differences; good results have been obtained also for thermographic image sequences [6].

In the present case the acquired sequence of $N$ infrared images ( $n_{x} \times n_{y}$ pixels) represents the source data volume to be process with the PCA algorithm.

The information contained in the original volume of data is both in space (defects geometry and location) and time (thermal contrast evolution). This volume is considered as a sequence of thermal contrast profiles and the previous defined matrix $A$ has $N$ rows (each column is a time profile for a pixel) and $n_{x} \times n_{y}$ columns. The data dimension is $m=N$ and the number of cases is $q=n_{x} \times n_{y}$.

Regarding the dimensions, the principal axes are temporal profiles and the projected data are images.

A software capable to execute the PCA and to structure the thermographic acquisitions in a suitable way for it have been developed in LabVIEW.

In this algorithm the PCA transformation from $m$-dimensional data to $p$-dimensional subspace was accomplished by choosing the first $p$ eigenvalues in such a way that the resulting amount of total variance is greater than 0.99 (i.e. the sum of first $p$ eigenvalues divided by $m$, representing the amount of total variance explained by the first $p$ principal components, is $>0.99$ ).

The results of PCA, having the same dimensions as an image, are called Spatial Components (SC). In each pixel of the Spatial Components, information on the whole thermal contrast evolution of that pixel are condensed, thus creating a set of very few (usually maximum $p=4$ in the present case) images representative of the entire sequence. In this set of images, information related to defect detection have been seen to be generally condensed in the first or second Spatial Component.

\subsection{Trend removal and automatic image threshold}

In defect detection methods based on analysis of thermographic images, the image background holds an important role; in fact, because of heating non-uniformity effects, the borders of the tile in an IR image may be sometimes hotter than the other parts.

This situation engenders some problems when an automated threshold algorithm has to be applied to a thermal image, as on the tile a clear separation between defects and background could not be achieved.

To solve this problem a procedure of trend removal (TR) has been implemented in LabVIEW and applied to raw or processed infrared images.

In this procedure a synthetic image was produced from the original image by making use of a two-dimensional polynomial fitting algorithm (below $7^{\text {th }}$ order) to obtain, after subtraction of the synthetic image by the original image, an uniform background on 
which defects appear more clearly (figure 3). The polynomial order has been always set below the $7^{\text {th }}$ order in such a way as not to mask as background small defects (e.g. $10 \mathrm{~mm}$ ) to be detected. In addition this order has shown to be enough to approximate the heating pattern of the employed test bench.

After the application of a Gaussian smoothing filter [7] with a $7 \times 7$ kernel, an automatic threshold technique, following the entropy method [8], was applied to separate the two modes (defects and background).

The Gaussian filter attenuates variations of the intensity in the neighborhood of a pixel to obtain a less noisy image; it is a smoothing filter where blurring effects are more subdued.

In contrast to manual thresholding, automatic thresholding algorithms do not require the setting of the minimum and maximum pixel intensities. These techniques are well suited for conditions of intensity variation in the image due to local differences in temperature or emissivity on the tile, as those due to local defects in the order of $10-$ $20 \mathrm{~mm}$, whose detection is the aim of the present work. In these situations it is impossible to identify fixed threshold values, as these would depend on the strength of the thermal effect of the defects, clearly a-priori unknown.

The entropy method is one of the most used for detecting particles that are present in minuscule proportions on the image and is suitable for fault detection. The threshold value is obtained by applying information theory [8] to the histogram data; in information theory, the entropy of the histogram signifies the amount of information associated with the histogram.

Finally the thresholded image have been eroded to eliminate pixels isolated in the background.

\section{Evaluation of the Contrast and Signal to Noise Ratio for different algorithms}

After the application of the processing techniques, a quantitative analysis of the influence of measurement uncertainty on the detection capabilities has been performed, in particular to evaluate NDT performances in relation to the noise and its propagation into the algorithms.

The calibration uncertainty in the infrared measurement, according to the thermal camera specifications, is $\pm 2{ }^{\circ} \mathrm{C}$ or $\pm 2 \%$ of the reading. However, for diagnostic purposes, the relevant information is connected to the gradients on the thermal image, and not on the absolute temperature value. Therefore the uncertainty in the detection procedure can be considered as mainly due to:

1. the noise, and thus the repeatability, in the measurement chain: this is mainly due to the sensor inherent noise (Noise Equivalent Temperature Difference $-\mathrm{NETD}=0.15^{\circ} \mathrm{C}$ ), but it is also affected by the repeatability and constancy in time of the whole measurement chain, i.e. of the following elements 2, 3 and 4 .

2. uniformity of the surface emissivity: this problem can be considered as negligible for the surface of a ceramic tile, which is flat and regular.

3. uniformity of the thermal excitation: in general it is very difficult (almost impossible) to have a full spatial homogeneity and in our case also the thermal excitation by the four lamps may have a not perfect uniformity over the tile surface. However, if repeatable, this can be dealt by post-processing algorithms (e.g. PPT or trend removal) as previously explained.

4. the measurement environment: it must be controlled that no other irradiating sources can affect the measured distribution. The environment can be 
considered under control for the tests performed in our laboratory, however, this point must be carefully evaluated for an on-line application.

It is possible to state that in this application points 2,3 and 4 must be considered mainly for their contribution to point 1 , i.e. the overall repeatability.

The repeatability of the whole measurement procedure has been therefore experimentally evaluated by repeated measurements at different times in the day, on different tiles and at different temperature levels. An average value of the temperature standard deviation was extracted for the whole image (as average of the standard deviation values estimated for each pixel in the different conditions) equal to $\overline{S_{n}}=0.25^{\circ} \mathrm{C}$. No image averaging was used in this case, as transient phenomena were measured.

At this point, in order to evaluate the NDT performances of the system and to compare the different post-processing algorithms, two parameters were defined and evaluated: a Signal to Noise Ratio $(S N R)$ of the thermal or processed image of the tile under inspection, see Eq. (3), and a Contrast to Noise Ratio (CNR) [9], calculated in a region of interest of the thermal image between amplitude values in the defect region and background values, see Eq. (4).

The $S N R$ was computed considering that for the single image the noise is characterized by its standard deviation $s_{n}$ and the signal is known to lie between two boundaries $\left(a_{\min }\right.$ and $\left.a_{\max }\right)$.

$$
S N R=20 \cdot \log _{10}\left(\frac{a_{\max }-a_{\min }}{s_{n}}\right)
$$

For the definition of the $C N R$ between the signal amplitude in defect zones and signal amplitude in the background, the knowledge of the defect position and extension was used to divide the image in two regions (defect and background).

Defining $\mu_{\text {defect }}$ and $\mu_{\text {back }}$ respectively mean amplitude values measured on the defect and background zones, similarly $\sigma_{\text {defect }}$ and $\sigma_{\text {back }}$ the standard deviations and $w_{\text {defect }}$ and $w_{\text {back }}$ the noise weights (as fraction of the total area, see Eq. (4)), the local CNR can be defined in Eq. (5).

$$
\begin{aligned}
& w_{\text {defect }}=\frac{\text { Area }_{\text {defect }}}{\text { Area }_{\text {defect }}+\text { Area }_{\text {back }}}, w_{\text {back }}=\frac{\text { Area }_{\text {back }}}{\text { Area }_{\text {defect }}+\text { Area }_{\text {back }}} \\
& C N R=\frac{\mu_{\text {defect }}-\mu_{\text {back }}}{\left[w_{\text {defect }} \cdot \sigma_{\text {defect }}^{2}+w_{\text {back }} \cdot \sigma_{\text {back }}^{2}\right]^{1 / 2}}
\end{aligned}
$$

These parameters were evaluated for raw thermographic data obtained by the IR camera, for phase maps from PPT and spatial components maps from PCA, after processing with trend removal and Gaussian smooth filtering. This allowed a quantitative comparison of the different approaches. 


\section{Analysis of results}

In this paragraph some results of thermographic acquisitions of defected tiles are shown; partial and final results are expressed as maps produced by TT, PPT, PCA, trend removal and automated thresholding techniques. As an example two tiles from the inspected series are here considered; their defects are constituted by plastic sheets placed at $5 \mathrm{~mm}$ depth and having different size (circular $15 \mathrm{~mm}$ for tile 1 and rectangular $30 \times 20 \mathrm{~mm}$ for tile 2 ).

The flowchart in figure 2 shows the alternative post-processing procedures: thermographic acquisitions of thermal decays on tile surfaces were employed to extract best raw thermograms and as inputs for TT, PPT and PCA algorithms.

Only a map was considered as output from PPT or PCA and the selection was done according to the maximum contrast between defect and background: in PPT this condition was verified always in the phase map at the first lower frequency band, while in PCA a selection was performed between different Spatial Components maps (but usually the first or the second ones were used).

Partial results in term of timegrams, phase maps, Spatial Components together with best raw thermograms have been then treated by removing the two-dimensional trend due to excitation pattern (trend removal technique, TR) and finally autothresholded.

Therefore estimations of $S N R$ and $C N R$ were accomplished on resultant maps not yet thresholded (in order to check real performances before separation in two discrete levels) and on the best raw thermogram with and without trend removal.

Analysis of results demonstrates that there is a good agreement (discrepancies below $10 \%$ in average) between real size of defects and area considered as defected by processing techniques. The employed set-up allowed a spatial resolution of 1.5 $\mathrm{mm}$ in the images.

Concerning the depth, it was observed that defects positioned below about $6 \mathrm{~mm}$ under the tile surface can not be detected, as the thermal effect is not measurable with the used set-up. Higher heating energy would be necessary to increase the inspection depth, as well as a higher resolution sensor. However, within the $6 \mathrm{~mm}$ depth under the surface, defects with dimension down to $5 \times 5 \mathrm{~mm}$ were identified, clearly with higher $C N R$ for those located closer to the surface.

In figures 3 and 4 examples of results are shown, illustrating main characteristics of the proposed processing algorithms. The raw thermograms, even if with high $S N R$, are almost not able to identify the defects. On the contrary, the defects are clearly detected after post-processing, even if with different performances depending on the approach (see Table 1).

Timegrams and spatial components are more sensitive to heating non-uniformities than phase maps; so the removal of the trend is necessary, particularly after tomography and PCA.

Furthermore thermal tomography makes impractical an automatic use (e.g. on line) because of its need of the definition of a sound area. On the contrary PPT and PCA are capable to concentrate in one (or very few) map the necessary information to detect the defected zone over the tile surface.

The results from Gaussian smooth filtering and auto-thresholding are reported in the last column, showing the capability in further separating the defected zones.

In average the highest performances in terms of $C N R$ and $S N R$ are given by the best raw thermogram taken in the acquired sequence combined with trend removal or by maps from PPT and PCA. However, the best raw thermogram needs an analysis of all the measured raw maps (e.g. 100 maps) to be identified, so it is less 


\section{http://dx.doi.org/10.21611/qirt.2006.003}

practically applicable. Therefore, overall it can be concluded that PPT and PCA are the more suitable for the application.

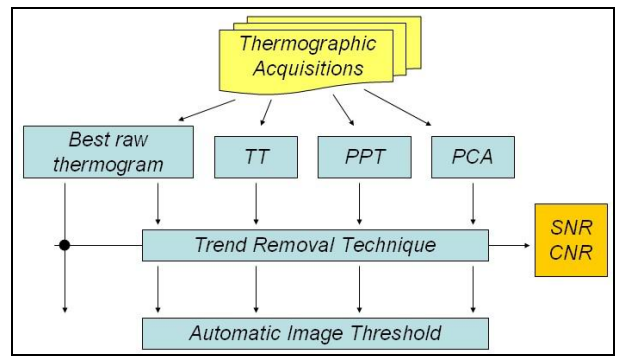

Fig. 2. Flow chart of the alternative adopted post-processing techniques; SNR and CNR calculations were done just after trend removed.

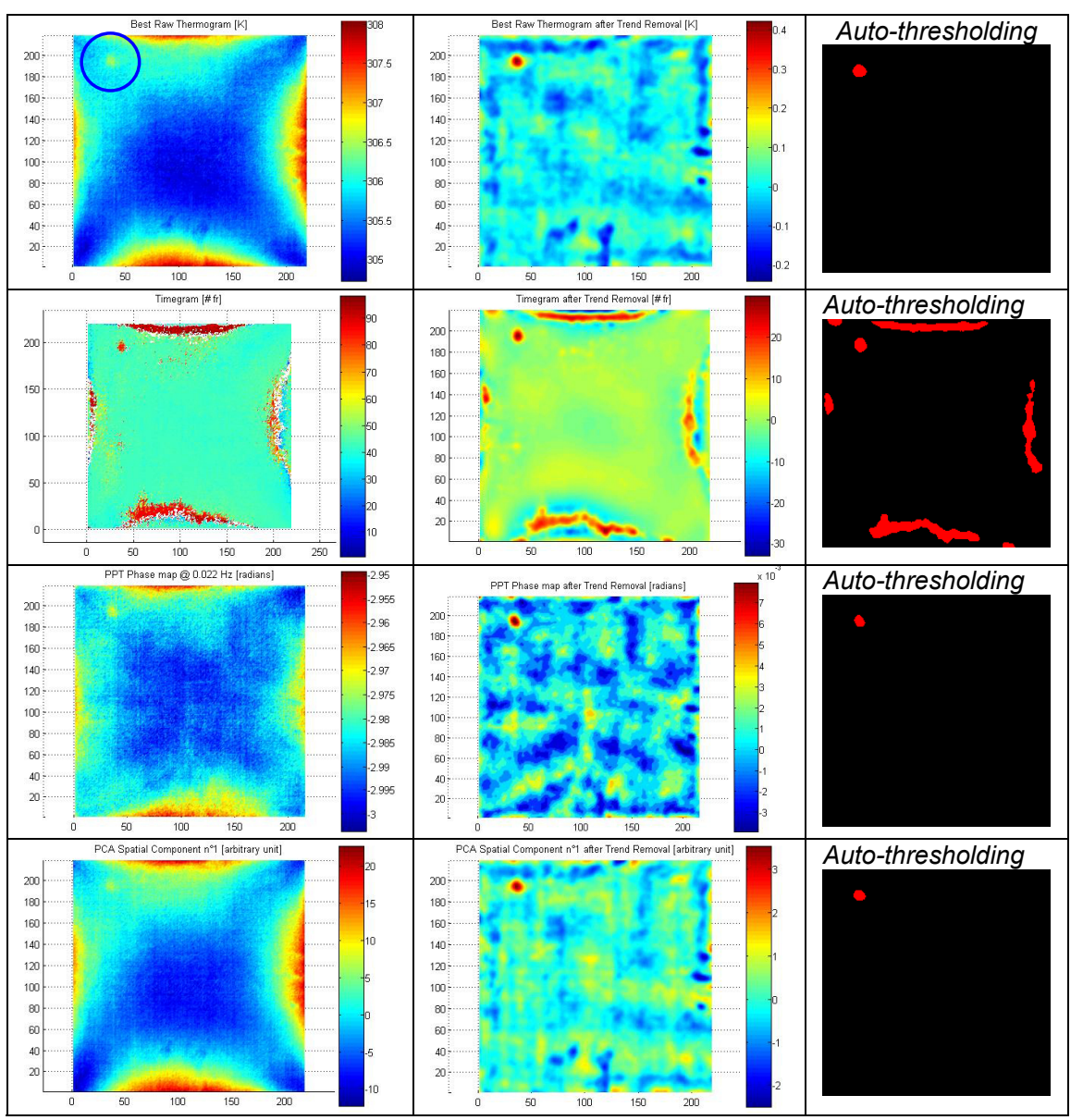

Fig. 3. Results for tile 1. 
http://dx.doi.org/10.21611/qirt.2006.003

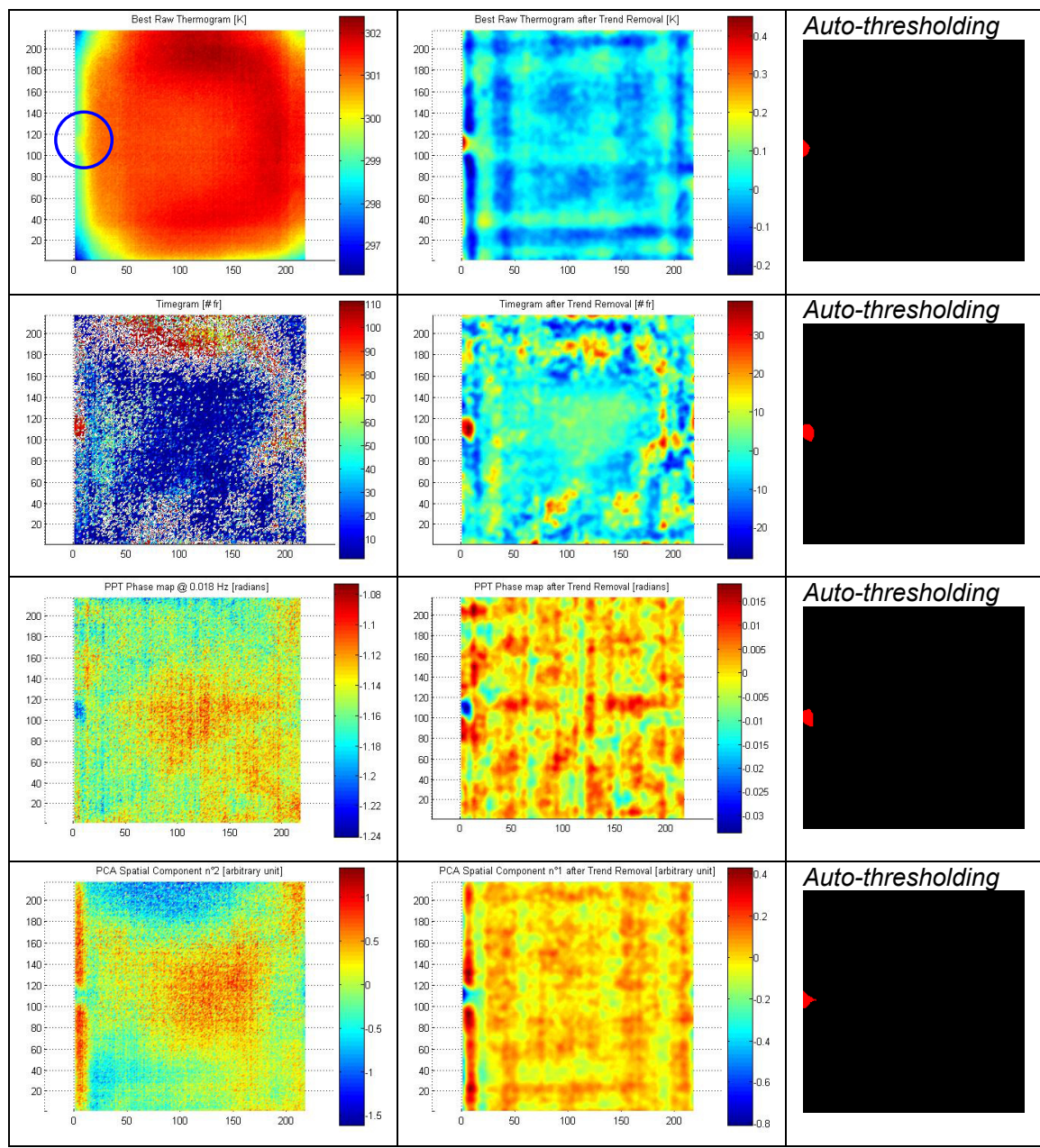

Fig. 4. Results for tile 2.

Table 1. Results of evaluations of $C N R$ and $S N R$ for two tiles and processing techniques.

\begin{tabular}{|l|c|c|}
\hline Input data & CNR & SNR [dB] \\
\hline Tile 1 - Best thermogram & 0.8 & 28.5 \\
\hline Tile 1 - Best thermogram + TR & 5.7 & 29.3 \\
\hline Tile 1 - Timegram + TR & 3.6 & 32.7 \\
\hline Tile 1 - PPT phase map + TR & 4.2 & 26.2 \\
\hline Tile 1 - PCA SC map + TR & 4.8 & 25.3 \\
\hline Tile 2 - Best thermogram & 1.6 & 35.6 \\
\hline Tile 2 - Best thermogram + TR & 2.6 & 30.3 \\
\hline Tile 2 - Timegram + TR & 2.7 & 31.4 \\
\hline Tile 2 - PPT phase map + TR & 3.4 & 22.4 \\
\hline Tile 2 - PCA SC map + TR & 3.6 & 27.3 \\
\hline
\end{tabular}




\section{Conclusions}

In this work a method for the detection of defects in ceramic tiles has been developed and applied. The method is based on two steps: 1) infrared thermal measurements during heating and cooling transients; 2) image processing to detect, locate and highlight the defect. The attention has been focused on the image processing. In particular different strategies (Thermal Tomography TT, Pulsed Phase Thermography PPT, Principal Component Analysis PCA) have been implemented and compared in terms of Signal-to-Noise and Contrast-to-Noise ratio, in order to identify the best procedure. Moreover also techniques to reduce the effects of non-uniform heating and to isolate the defects from background were considered.

Results indicated that generally the application of PPT and PCA algorithms enhances the gap between defect and background, maintains almost constant the SNR of the map and improves applicability. As an example, defects of dimension $15 \mathrm{~mm}$ located $6 \mathrm{~mm}$ below the surface were detected with a $S N R$ higher than $25 \mathrm{~dB}$ and a $C N R$ higher than 4 .

\section{ACKONWLEDGMENTS}

Part of this research was carried out within the project MONOTONE, financed by the European Commission in the 5th Frame Programme (Growth Programme, G1RD-CT 2002-00783). The author would like to thank the partner Leonardo 1502 Ceramica of Casalfiumanese (BO) for the preparation of the samples.

\section{REFERENCES}

[1] R. M. De Andrade, N. Paone, G. M. Revel, Non Destructive Thermal Detection of Delamination in Ceramic Tile, Proc. ENCIT 98, Rio de Janeiro, (1998) 727731.

[2] R. M. De Andrade, E. Esposito, N. Paone, G. M. Revel, Non-destructive Techniques for Detection of Delamination in Ceramic Tiles: a Laboratory Comparison between IR Thermal Cameras and Laser Doppler Vibrometers, Proc. SPIE Vol. 3585, p. 367-377, Non-destructive Evaluation of Aging Materials and Composites III, G. Y. Baaklini; C. A. Lebowitz; E. S. Boltz; Eds (1999).

[3] X. P. V. Maldague, Theory and Practice of Infrared Technology for Nondestructive Testing, John Wiley \& Sons, New York, NY (2001).

[4] X. P. V. Maldague, S. Marinetti, Pulse Phase Infrared Thermography, Journal of Applied Physics, 79[5] (1996) 2694-2698.

[5] I. T. Jolliffe, Principal Component Analysis, Springer-Verlag, New York (1986).

[6] S. Marinetti, E. Grinzato, P. G. Bison, E. Bozzi, M. Chimenti, G. Pieri and O. Solvetti, Statistical analysis of IR thermographic sequences by PCA, Infrared Physics and Technology, 46 [1, 2], Elsevier (2004).

[7] IMAQ Vision Concepts Manual, NI Vision, National Instruments (2005).

[8] J. N. Kapur, P. K. Sahoo and A. K. C. Wong, A new method of gray level picture thresholding using the entropy of the histogram, Computer Vision, Graphics and Image Processing, 29 (1985), 273-285.

[9] X. Song, B. W. Pogue, S. Jiang; M. M. Doyley, H. Dehghani, T. D. Tosteson, K. D. Paulsen, Automated region detection based on the contrast-to-noise ratio in near-infrared tomography, Applied Optics, 43 [5] (2004) 1053-1062. 